Cinémas

Revue d'études cinématographiques

Journal of Film Studies

\title{
De la réception des films au cinéma des ouvriers
}

\section{Fabrice Montebello}

Volume 2, numéro 2-3, printemps 1992

Cinéma et Réception

URI : https://id.erudit.org/iderudit/1001081ar

DOI : https://doi.org/10.7202/1001081ar

Aller au sommaire du numéro

\section{Éditeur(s)}

Cinémas

ISSN

1181-6945 (imprimé)

1705-6500 (numérique)

Découvrir la revue

Citer cet article

Montebello, F. (1992). De la réception des films au cinéma des ouvriers.

Cinémas, 2(2-3), 123-147. https://doi.org/10.7202/1001081ar

\section{Résumé de l'article}

L'étude des usages sociaux du cinéma permet de rompre avec la thématique des effets de ce dernier sur le ou les spectateurs. Elle considère les films comme des biens symboliques, à la fois objets et enjeux de distinctions sociales.

L'histoire de l'usage social du cinéma (qui a été populaire avant d'être un art) fait apparaître la formation d'un code de lecture populaire des films, spécifique et autonome, parallèlement à la constitution d'une esthétique bourgeoise légitime. L'analyse des catégories employées pour justifier l'adhésion à tel ou tel film, révèle alors un univers symbolique ouvrier original, et suscite des perspectives nouvelles pour l'histoire des " cultures populaires ». Ce texte présente quelques remarques méthodologiques et hypothèses de recherche sur une micro-histoire des usages populaires du cinéma : l'exemple des ouvriers de Longwy (1945-1960). 


\title{
De la réception des films au cinéma des ouvriers
}

\section{Fabrice Montebello}

\author{
RÉSUMÉ
}

Létude des usages sociaux du cinéma permet de rompre avec la thématique des effets de ce dernier sur le ou les spectateurs. Elle considère les films comme des biens symboliques, à la fois objets et enjeux de distinctions sociales. L'histoire de l'usage social du cinéma (qui a été populaire avant d'être un art) fait apparaître la formation d'un code de lecture populaire des films, spécifique et autonome, parallèlement à la constitution d'une esthétique bourgeoise légitime. L'analyse des catégories employées pour justifier l'adhésion à tel ou tel film, révèle alors un univers symbolique ouvrier original, et suscite des perspectives nouvelles pour l'histoire des «cultures populaires». Ce texte présente quelques remarques méthodologiques et hypothèses de recherche sur une micro-histoire des usages populaires du cinéma: l'exemple des ouvriers de Longwy (1945-1960).

\section{ABSTRACT}

The study of the social uses of cinema makes it possible to break with the old theme of its effects on its spectators. It views films as symbolic goods which are both the objects of and the stakes in social distinctions. The history of the social use of cinema (a form that was popular before it became art) highlights the emergence of a folk code for reading films. This specific autonomous code appeared in parallel with the development of the legitimate bourgeois film aesthetic. An analysis of the 
categories invoked to justify a preference for such and such a film reveals a specific working class symbolic universe and thus opens new perspectives on the history of popular cultures. This article presents methodological comments and research hypotheses using the example of a micro-history of popular uses of film among the workers of Longwy (1945-1960).

Dans l'étude suivante, nous avons volontairement écarté de nos préoccupations des approches qui, à partir du film seul comme corpus, et sous l'influence de disciplines diverses (linguistique, sémiologie, psychanalyse, sémio-pragmatique, etc.), font du spectateur une instance théorique, sans jamais le définir historiquement ou sociologiquement ${ }^{1}$. Le cadre monographique ${ }^{2}$ proposé ne doit pas être entendu uniquement comme une concession méthodologique à une problématique qui se révélerait invérifiable pour un ensemble plus vaste, comme un pays par exemple. L'attention portée à des micro-événements, structurant dans le passé comme dans le présent la vie du groupe ouvrier étudié (pas plus de six personnes), nous a permis de mettre en valeur des hypothèses de recherche et des questions qu'une vision plus éloignée contribuait à masquer ${ }^{3}$. Dans cet article, l'étude de la réception des films n'est jamais considérée comme une fin en soi, mais comme un champ nouveau qui s'ouvre à l'historien des mentalités dans l'appréhension d'un «style de vie» populaire ${ }^{4}$.

\section{Les études sur le public de cinéma}

Les études portant sur le public de cinéma sont à la fois nombreuses et très anciennes. Elles sont nées en général de la croyance dans la force de persuasion du réalisme photographique qui a contribué à centrer les problématiques des rapports entre cinéma et public autour de la question des effets de ce dernier sur le ou les spectateurs ${ }^{5}$. Il n'est pas difficile de ramener de telles préoccupations aux contextes économiques (fordisme, taylorisation), idéologiques (suffrage universel, avènement et affrontements de régimes totalitaires modernes) ou culturels (la culture légitime comme culture aristocratique des élites) qui caractérisent la première moitié du $\mathrm{XX}^{\mathrm{e}}$ siècle. Les études oscillent alors entre la dénonciation du cinéma et de ses effets en 
termes de «manipulation» ou d' «aliénation» ${ }^{6}$.

$\mathrm{Au}$ point de vue aristocratique des «masses dégradant l'art» répond l'idée des avant-gardes de gauche selon laquelle il faut politiser l'art ou de manière plus ambiguë encore il faut éduquer les masses, les sensibiliser au jugement de goût ${ }^{7}$. Même si les analyses se sont affinées depuis, les présuppositions qui font des spectateurs des êtres passifs recevant sans résistance des messages et subissant leur influence, demeurent prégnantes, surtout lorsque ces spectateurs proviennent des classes sociales subalternes que l'on associe systématiquement à l'indigence critique et à la pauvreté intellectuelle. La théorie de la légitimité culturelle transparaît alors dans les discours et assimile les pratiques culturelles populaires à l'expression d'un goût dominant dégradé (euphémisme de «mauvais goût»).

Il est vrai que l'étude de la réception cinématographique pose des problèmes méthodologiques considérables. Comment mesurer ou recueillir les données qui règlent les modalités de la perception des films au cinéma? Comment, pour reprendre une question simple, regardait-on un film dans les années 40-50 au cinéma ${ }^{8}$ ? La question est plus cruciale encore lorsque l'on s'est fixé pour tâche de l'appréhender au sein des classes populaires. Si le chercheur peut se rendre à l'évidence d'une forte présence populaire dans les salles de cinéma au cours des années qui suivent l'immédiat aprèsguerre ${ }^{9}$, il n'en demeure pas moins dans l'incapacité, étant donné la faible propension de ces classes à témoigner spontanément de leurs impressions ou sentiments, d'en retrouver les motivations profondes.

La tendance à penser le «public» comme une instance séparée, en dehors de la relation qu'il entretient avec les films, renforce cette incapacité méthodologique et renvoie à nouveau la question du spectateur à une problématique en termes d'«effets». On homogénéise alors la réception des films à partir de leurs diffusions et l'on infère des qualités du film, les caractéristiques des spectateurs censés le regarder; comme dans l'expression «cinéma populaire», où l'on semble postuler l'existence d'un genre cinématographique à part entière, autonome, possédant ses propres règles de production et de création et capable de répondre aux attentes d'un public supposé populaire. C'est en modifiant à la 
fois l'approche méthodologique traditionnelle, les questions que l'on se pose habituellement à propos du public de cinéma et l'objet même de ces questions, que nous tenterons de présenter nos hypothèses de recherche.

\section{L'usage social du cinéma comme objet d'étude}

Une première rupture consisterait à s'interroger sur le rapport que le chercheur entretient avec son objet d'étude, de manière à éviter tout risque d'intellectualisme que Pierre Bourdieu définit comme «le fait d'introduire dans l'objet le rapport intellectuel à l'objet, de substituer au rapport pratique à la pratique le rapport à l'objet qui est celui de l'observateur», de «faire comme si les agents se posaient les questions que le chercheur se pose à leur propos» (1980, p. 53). Ainsi, l'usage de catégories dégagées de leur «principe de constitution ${ }^{10}$ » amène l'observateur à disserter à propos d'une réalité historique dont certains enjeux lui échappent, comme lorsque Patricia Hubert-Lacombe se sert du paramètre «fréquentation des films américains» pour mesurer la force de pénétration de l'impérialisme idéologique des États-Unis en France au lendemain de la Seconde Guerre mondiale, oubliant du même coup ce que peut signifier aller voir un film américain pour nombre de contemporains de l'époque ${ }^{11}$. À Longwy, par exemple, comme nous le verrons plus loin, l'expression «film américain» représente, pour une certaine fraction du public ouvrier, un cri de ralliement esthétique (comme d'autres parlent de «cinéma d'auteurs») qui ne relève pas d'antagonismes idéologiques mais traduit tout à la fois des formes de jugements autonomes et des enjeux de distinction sociale.

Ces remarques présupposent qu'on ne saurait dissocier la diffusion d'un message culturel des conditions sociales de sa réception. Les différents individus qui composent le public ne reçoivent pas les messages comme des ordres donnés à de simples automates, mais les trient en fonction de leur vision du monde et des principes qui règlent les actes de leur vie quotidienne, euxmêmes déterminés par leurs conditions sociales d'existence. Richard Hoggart parle ainsi d' «attention oblique» ou d' «adhésions à éclipses» pour caractériser la capacité des membres des classes populaires, les plus démunis culturellement et donc les plus 
enclins selon les présupposés précédents à la manipulation ou au conditionnement, à filtrer les messages culturels en fonction de leur philosophie de la vie ${ }^{12}$. C'est pourquoi on ne saurait parler de «cinéma populaire», mais d'usages populaires du cinéma. Un tel postulat implique également une acception plus large de ce que l'on entend généralement par «réception d'un film». Voir un film est un phénomène complexe qui ne se réduit pas au seul fait de le regarder dans une salle de cinéma pendant un certain temps, mais de se le remémorer, d'en parler autour de soi, de préparer la prochaine sortie. Il s'agit donc pour le chercheur de prendre en considération l'avant et l'après, d'analyser la somme des actes et des investissements dont les films sont l'objet de la part des spectateurs.

Les modalités de cette «réception» prennent alors des allures diverses selon la période historique considérée, le lieu géographique étudié, l'organisation de l'espace urbain, la répartition des salles, leur intégration dans les quartiers et leur cotation officielle. Par exemple, dans les années 40-50, les salles étaient classées en catégories officielles A, B, C, ou D, selon des critères techniques allant de l'existence d'un balcon à la qualité des sièges ou de l'appareil de projection et à celle de l'insonorisation ${ }^{13}$. À cet espace réel se superpose un univers symbolique dans lequel joue une autre classification qui renvoie à la composition sociologique de certaines salles ou plutôt à l'image que l'on s'en fait «salle populaire», «cinéma rupin», «cinéma des curés», etc. Mais les déterminations les plus importantes concernent les agents sociaux dont l'attitude à l'égard du cinéma dépend non seulement de la situation sociale ou professionnelle, mais également de l'âge, du sexe, de l'héritage de dispositions familiales particulières, de l'appartenance à des nationalités ou des ethnies différentes, enfin des relations que les spectateurs habitués (ou non) entretiennent avec le propriétaire ou le gérant de la salle. Ajoutons, à ces facteurs qui orientent la nature du regard, la pratique cinématographique elle-même: sorties collectives («entre copains» ou en famille), sorties en couples ou individuelle, fréquentation «rituelle», suivie ou spécialisée (cinéphilie), lectures de journaux ou revues sur l'actualité cinématographique, prolongement de la soirée dans la rue ou au café, etc. 


\section{Logiques de distinction sociale et limites du relativisme culturel}

La particularité qui caractérise le cinéma ne tient donc pas seulement à sa nature (ce spectacle «riche de suggestions directes» ${ }^{14}$ ) mais à sa fonction sociale qui fait de lui, comme de tout autre bien culturel, l'objet et l'enjeu d'une distinction sociale entre les agents d'une société. Cette distinction ne s'opère pas uniquement selon les différents objets symboliques appropriés (ici les films ou types de films) mais également selon les différents modes d'appropriation de ces objets ${ }^{15}$. Ainsi, à Longwy, le souvenir d'un plaisir cinématographique ritualisé, dans lequel intervient le cinéma comme métaphore religieuse («regarder religieusement un film») rappelé par un ancien animateur de cinéclub, s'oppose à la «mémoire» ouvrière d'une jouissance anarchiste des films s'accomplissant dans le tumulte et la confusion de voix et de commentaires intempestifs ${ }^{16}$.

Le cinéma dans les années 40-50, loin d'être considéré comme un art et une pratique culturelle noble, voit l'émergence de discours de légitimation nombreux et parfois contradictoires, tentant de distinguer à l'intérieur de ce que tous considèrent comme le «Septième Art», le bon cinéma du mauvais. Les discours émanant de groupes ou d'institutions différentes s'accompagnent de la tentative de mise en œuvre de catégories de jugement nécessaires à l'appréciation de la valeur des films et de leur hiérarchisation. Le Parti communiste français met ainsi en avant des justifications de type politique ou correspondant à une certaine réalité sociale, l'Église favorise la promotion de films véhiculant les messages chrétiens. Quant aux ciné-clubs naissant, s'ils sont les plus enclins à rechercher des critères de jugement autonomes, ils restent marqués par un souci de didactisme et de «bonne volonté culturelle» (discours sur les «classiques du cinéma» et la promotion des films à l'égal des grandes œuvres artistiques) ${ }^{17}$. Si toutes ces «esthétiques en formation» ont en commun le rejet des usages populaires du cinéma (associés au divertissement naïf) et celui de l'image des films correspondants (identifiés à des logiques commerciales), il faut attendre la professionnalisation de certains secteurs, la transformation d'intellectuels d'institutions (comme les animateurs de ciné-clubs par exemple) en journalistes 
critiques de cinéma possédant un statut spécifique, l'exercice de ce métier au sein de revues spécialisées et indépendantes ( $L$ 'Écran français, La Revue du cinéma, Les Cahiers du cinéma ${ }^{18}$, etc.), en un mot ce que Pierre Bourdieu appellerait l'autonomie du «champ des intermédiaires cinématographiques», pour voir l'apparition d'un code de lecture des films légitime et autonome.

Tout le monde s'accorde aujourd'hui à voir dans le réalisateur l'«auteur» du film, la figure de l'«artiste» puisant dans son inspiration les forces nécessaires à la «création de l'œuvre». On repère les filiations d'école (l'influence du néo-réalisme ou des formalistes russes), les héritages cinéphiliques («c'est du Mizoguchi», «c'est du Welles», "c'est du Renoir», etc.), les grandes ruptures du «Septième Art» (Rossellini et la naissance du "cinéma moderne»). Le vocabulaire de la grâce élude ainsi la prise en considération des conditions historiques et sociales de production et de réception des films et est à l'origine d'une mémoire cinéphilique commune que vient à son tour légitimer le discours universitaire.

Cependant, si le relativisme culturel de Bourdieu permet de ne plus dissocier l'objet de son usage social (ici le film des conditions de sa réception) et donc d'en finir avec la valeur intrinsèque de l'œuvre d'art, de révéler les mécanismes sociaux qui fondent le jugement esthétique, il n'en demeure pas moins marqué par une vision par le haut de ces mêmes mécanismes et reste prisonnier d'une problématique en terme de «légitimation» qui attribue de moins en moins de liberté ou de choix aux individus, au fur et à mesure que l'on descend dans la hiérarchie sociale.

Ce type de généralisation introduit alors dans l'observation d'une esthétique, des critères eux-mêmes d'ordre esthétique (par exemple dans l'emploi des couples de contraires «en soi»/«pour soi», «forme»/«contenu», «goût de nécessité»/«goût de liberté», où les premières caractéristiques sont bien évidemment du côté des classes dominantes et les secondes, du côté des classes populaires ${ }^{19}$ ). Elle fait abstraction du terrain particulier sur lequel se mesure la compétence culturelle (comme ici le cinéma) et sousestime la part d'appropriation ou de reconstruction des messages culturels de la part du spectateur des classes populaires. Elle réduit les goûts des classes dominées à des choix mécaniques et 
prévisibles $^{20}$. Le déterminisme sociologique de Pierre Bourdieu se transforme en "prédestination sociale» où l'on devine des produits populaires par anticipation des goûts correspondants (logique de la réponse à l'attente supposée) ${ }^{21}$. À l'épreuve d'une vision rapprochée des choses qui offre à l'observateur des relations sociales et des points de vue à la fois multiples et divers, cette dernière démarche tend à déboucher sur des apories. Elle incline également à penser la «culture du pauvre» comme culture «plus pauvre».

Or on peut supposer des formes d'appropriation de biens symboliques qui ne se réduisent pas uniquement à des logiques de distinctions sociales. Le cinéma d'immédiat après-guerre n'est pas seulement ce terrain particulier sur lequel s'affrontent des discours de légitimation contradictoires comme nous l'écrivions précédemment; il est également le lieu où s'exerce, par l'intermédiaire d'une longue familiarité et d'une expérience pratique, une compétence populaire spécifique et réelle. Resitués dans une pratique culturelle générale qui n'a toujours pas trouvé de consécration en 1950, les usages populaires du cinéma peuvent se vivre dans l'oubli de ces enjeux de distinction et devenir «le lieu privilégié d'activités culturelles les moins marquées par les effets symboliques de la domination» (Grignon et Passeron, p. $81^{22}$ ). D'autre part, subordonner complètement l'appropriation de biens symboliques à l'héritage de schèmes sociaux de classifications (l'habitus), comme le fait Pierre Bourdieu, empêche de dissocier une opération complexe comme celle du jugement des conditions historiques et sociales qui le déterminent, et oublie de le ramener au cadre matériel et symbolique dans lequel se déroule l'expérience esthétique. En d'autres termes, si l'habitus influence le jugement esthétique en déterminant notamment la familiarité avec tel ou tel bien culturel et les usages légitimes dont il est l'objet (la fameuse «disposition esthétique»), il ne se substitue pas ni ne se confond avec lui. Le cinéma, au même titre que d'autres biens symboliques, appelle la mise en œuvre d'outils cognitifs particuliers qui ne sont pas déterminés par les conditions sociales de réception des films, mais par le cinéma lui-même. Ainsi, la vision d'un film commande la mise en œuvre d'outils cognitifs spécifiques (ce que d'autres appelleraient «consignes de lecture»), mais se traduit par 
des usages sociaux multiples. Il ne s'agit donc pas pour le chercheur de ramener des préférences populaires à des films correspondants ou des conditions sociales générales à des goûts particuliers, mais d'individualiser les éléments mis en avant par les membres des classes populaires pour justifier l'adhésion à tel ou tel film.

\section{Principes de justification et codes de lecture: la question de l'accord}

L'ensemble de ces assertions appelle des définitions plus précises que nous illustrerons par des exemples concrets. Lorsque nous parlons de jugement, nous entendons une aptitude (que nous reconnaissons à tous les agents) à comparer, autrement dit à mettre de l'ordre, qui ne se résume pas à la seule capacité de formaliser explicitement ou rationnellement une opinion. La larme qui coule sur la joue d'un spectateur «bouleversé» par la projection d'un film, le cri que peut pousser un enfant pour accompagner le bruit de tam-tams d'indiens prêts à se lancer à l'assaut d'une garnison, la remarque «insignifiante» d'un ouvrier selon laquelle il s'agit d' «un beau film avec de bons acteurs», témoignent d'un rapport particulier au film qui est tout à la fois le résultat d'un ensemble de constructions sociales (pleurer, ressentir une émotion, «se justifier») et la manifestation d'ûn «penchant», d'un «goût», d'un jugement, c'est-à-dire l'expression implicite d'un principe de justification. Cette opération ne se distingue en rien de celle de l'intellectuel, qui après avoir ressenti une émotion tout aussi forte bien que différente, couchera sur le papier, de manière explicite, les catégories plus ou moins complexes qui servent d'illustration à son principe de justification. Certes, dans les sociétés de classes différenciées socialement, on distinguera bien évidemment, à propos des points de vue émis, des degrés de formalisation plus ou moins complexes. Mais dans l'usage que fait un agent, quel qu'il soit, d'un film, il y a toujours un jugement, et donc, un principe de justification. Ce dernier renvoyant implicitement l'objet observé à un système de classification plus complexe mais cohérent. En paraphrasant une célèbre citation de Gramsci, nous pourrions dire que tous les hommes sont des esthéticiens.

Il nous faut maintenant essayer de comprendre dans quelle 
mesure un principe de justification, qui est essentiellement une caractéristique individuelle, peut être partagé par plusieurs personnes. En d'autres termes, il s'agit de comprendre comment se fait l'accord sur la vision d'un film et donc sur son jugement. L'attitude du public dans les salles de cinéma, à des lieux et des époques différentes, témoigne de quantité de manifestations de reconnaissance collective: piaffer d'impatience au moment où les lumières s'éteignent, rire ou pleurer ensemble, siffler ou protester, maugréer à la sortie de la salle, rencontrer des amis avec lesquels on partage un plaisir commun, etc. Mais des dissensions peuvent également apparaître et témoigner de «fractures» à l'intérieur du public. Telle personne demandera: «un peu de silence s'il vous plaît», d'autres mettront en avant des justifications plus tranchées: «dans une salle de cinéma, on ne fait pas de bruit», ou encore: «si le film ne vous intéresse pas, vous pouvez sortir». Dans les années 40-50, les comportements dans les salles de cinéma étaient extrêmement bruyants. Il apparaissait alors normal de souligner son plaisir, son adhésion au film ou son rejet, en applaudissant, en sifflant, en parlant haut et fort pour commenter les scènes ou encourager les «héros», mais également en mangeant ou en fumant. Les cinémas étaient des lieux de convivialité qui ne se limitaient pas au seul plaisir cinématographique, même si ce dernier était systématiquement associé à la fête ${ }^{23}$. La drague, les farces, la joie de se retrouver entre amis étaient chose commune et le mieux partagée. Il est vrai que ces types de comportements apparaissaient plus facilement dans les salles populaires; mais comme le cinéma, dans les années d'immédiat après-guerre, était un fait majoritairement populaire, il ne serait pas étonnant de retrouver des caractéristiques similaires dans la majorité des salles de l'époque. On serait ainsi tenter d'analyser ces comportements comme des manifestations d'adhésion naïve aux films, révélatrices d'une «esthétique populaire» que Bourdieu définirait comme une «esthétique annexée à l'éthique» (1982, p. V et VI), «fondée sur l'affirmation de la continuité de l'art et de la vie qui implique la subordination de la forme à la fonction» (1982, p. 57). Ce serait penser que ces manifestations renvoient uniquement au film, à son histoire, à son contenu et non au jugement porté sur lui. Les applaudissements dans les salles 
populaires d'hier ne se distinguent pourtant en rien de ceux que délivre régulièrement aujourd'hui, au sein de la cinémathèque française, la «tribu» des cinéphiles, à ces films ou scènes de films qu'elle considère comme des classiques. Dans les deux cas, le geste ne s'adresse évidemment pas aux «fantômes de l'écran», mais au public de la salle qui partage le même plaisir dans la reconnaissance de mêmes valeurs.

Le passage d'un point de vue individuel à une manifestation de reconnaissance collective se traduit par la cristallisation de principes de justification en code de lecture. Ce dernier peut se définir comme un système de catégories de jugement (le plus souvent explicite) attribuant aux films une valeur reconnue par l'ensemble d'un groupe et le classant au sein d'une hiérarchie commune, c'est-à-dire communément admise. Le code de lecture est l'objet d'une construction collective qui justifie alors l'existence du groupe comme une «communauté d'interprétation» des films. Dans ce tout que forme un public de cinéma existent autant de principes de justification qu'il y a de spectateurs. Ceux-ci se constituent en groupe selon l'affinité des principes avec le code de lecture correspondant. Les tensions et les dissensions apparaissant à l'intérieur d'une même salle s'expliquent par la coexistence de codes de lecture opposés qui traduisent la confrontation d'univers esthétiques différents. L'esthétique, essentiellement définie comme une «activité et non comme un corps de doctrines ${ }^{24}{ }^{\text {», }}$ est donc ce geste qui consiste à classer des biens symboliques (films, livres, peintures, etc.) dans les registres du «beau» ou du «laid» et à le justifier. À justifier ce geste, c'est-à-dire à exprimer un jugement et, dans une certaine mesure, l'expliciter, le formaliser. Un geste qui consiste à «dire»: ce film est digne d'être vu pour moi, comme pour le groupe auquel j'appartiens et qui y reconnaît la même valeur.

\section{L'exemple des ouvriers de Longwy}

Au lendemain de la Seconde Guerre mondiale, à Longwy, l'abandon progressif de la fréquentation des cinémas de quartier situés à la périphérie de la ville, au profit de ceux du centre, traduit le passage de jeunes générations ouvrières à l'âge adulte. L'entrée dans le monde du travail se conjugue avec une émancipation 
progressive de la tutelle familiale, des parcours urbains inédits, de nouveaux lieux et modes de sociabilité (cinéma, bal, café...), la découverte d'expériences «interdites» (tabac, alcool, liberté sexuelle...). Le cinéma qui cristallise bon nombre de ces interdits peut se lire également comme le prolongement de cette culture ouvrière de la rue. La salle, Le Cinéma des Familles, dénoncée par la petite bourgeoisie locale comme le repaire de la «racaille» est, durant ces années, un haut lieu de rassemblement de la jeunesse ouvrière. On y compense l'inconfort général de la salle (fauteuils en bois, acoustique désastreuse, mauvaises odeurs...) par la joie de se retrouver ensemble (entre ouvriers, «entre nous») et une liberté de comportement inconnu ailleurs (on fume, on mange, on drague, on hurle, on commente le film et encourage les acteurs/ héros). Les conditions même de visionnement des longs métrages supposent un plaisir et un usage collectif des films, là où le cinéma «bourgeois» de la ville, Le Palace, impose une tenue stricte, un silence quasi religieux, en accord avec les représentations dominantes de l'art qui suppose le recueillement et un rapport individuel à l'œuvre et à son créateur.

Cette nouvelle vision du cinéma s'impose progressivement, notamment par l'intermédiaire des ciné-clubs qui apparaissent à Longwy dès 1949. Ils sont animés par la petite bourgeoisie locale (cadres, instituteurs, professeurs, employés) ${ }^{25}$ et prolongent toute cette entreprise de moralisation du cinéma menée par des sociétés liées à l'Église catholique ou des associations de «cinéma pour enfants». De leur côté, les organisations ouvrières de la région, comme le Parti communiste français (PCF) et le syndicat qui en est très proche, la Confédération générale du travail (CGT), proposent au cours des soirées de propagande des films édifiants d'un «très grand intérêt pour la classe ouvrière» ${ }^{26}$. Toutes ces instances ont en commun le rejet des films américains pour des raisons à la fois esthétiques («le cinéma de l'impérialisme»), ou morales («le cinéma, c'est le mal»).

Au même moment, pourtant, Le Cinéma des Familles, cette salle ouvrière qui ne désemplit pas, ne diffuse pratiquement que des films américains ${ }^{27}$. Ignorant tout de ces enjeux de distinction, les jeunes ouvriers qui s'autodésignent comme «amateurs de cinéma», construisent leur propre univers esthétique. La parole est 
certes fugitive et les rencontres informelles, pourtant, au fil des discussions impromptues dans la rue, les cafés, avant ou après la séance, pendant la projection, voire au sein même des ateliers d'usine, des points de vue s'échangent, des avis de confrontent, des accords se forment et se traduisent dans les admirations et les rires communs. Un groupe se constitue avec ses leaders, ses spécialistes, ses taste-makers.

«Alors on voyait à peu près, quand on voyait un western, on disait on y va, ça y a pas d'problème, surtout (rires) comme disait l'Bébert quand y'avait des "grands noms", lui i' nous guidait des fois, "y a un beau film par là, suivez-moi" qu'i' disait, hop nous voilà partis" (témoignage de Monsieur R.G., né en 1930, ancien ouvrier sidérurgiste).

Il est difficile pour l'historien de dégager à propos d'une microculture orale des lignes de force aussi nettes que celles qui se stabilisent dans des écrits ou se déposent dans une histoire légitime. Selon notre hypothèse de départ, il n'existe pas de genres cinématographiques a priori, mais des reconstructions de séries a posteriori, des appropriations après coup. Notre pari est donc de retrouver dans les témoignages et les dispositions des individus d'aujourd'hui, non pas l'image dẽformée d'une passion oubliée, mais les réminiscences d'un plaisir partagé. La force et la cohérence du souvenir devant révéler l'ancien degré de cohésion du groupe, sa capacité à mettre en avant des principes de justification communs. Ce qui nous apparaît alors, c'est une critique en actes et en paroles du cinéma, un point de vue original et spécifiquement populaire.

On remarquera que le groupe en question se distingue de celui des militants ouvriers qui connaissent, au même moment, de par leurs fonctions syndicales ou politiques, un processus d'acculturation et développent une «esthétique petite bourgeoise» du cinéma proche de celle des ciné-clubs.

Dans l'analyse des données relevées à Longwy, la vision ouvrière des films est fortement corrélée à un principe de justification qui renvoie à un monde et une logique industriels. Les jugements, entendus au sens où nous les avons précédemment définis, hiérarchisent les films en fonction de leur «efficacité», efficacité de l'histoire comme de la mise en scène, qui témoignent 
à leur tour de l'efficacité d'un cinéma considéré comme la référence obligée et incontournable: le cinéma américain. Le vocabulaire employé fait également référence à la «professionnalisation», la «compétence», la «démesure technique», la «performance». Toutes ces catégories ne correspondent pas à des genres préétablis (les grands spectacles hauts en couleur, les comédies musicales, les grandes reconstructions historiques, les films de guerre ou d'aventure, etc.), mais à des ensembles de films qui sont considérés comme des «classiques» et à partir desquels on compare d'autres films. Le «film américain» est la mesure étalon de la forme cinématographique par excellence; tout ce qui s'y rattache, «c'est du cinéma». Le «film français» en représente l'envers absolu, le «non-film», le «non-cinéma».

Citons avec prudence un certain nombre de films qui reviennent le plus souvent dans ces entretiens ou observations: Sergent York (Sergeant York) d'Howard Hawks (USA, 1941), Le Grand Sommeil (The Big Sleep) d'Howard Hawks (USA, 1946), Le Faucon maltais (The Maltese Falcon) de John Huston (USA, 1941), La Poursuite infernale (MyDarling Clementine) de John Ford (USA, 1946), Tant qu'il y aura des hommes (From Here To Eternity) (USA, 1953), La Maison des otages (The Desesperate Hours) de William Wylar (USA, 1955), Nous avons gagné ce soir (The Setup) de Robert Wise(USA, 1949), Aventuresen Birmanie (Objective Burma) de Raoul Walsh (ÜSA, 1945), Attaque (Attack) de Robert Aldrich (USA, 1956), etc. On n'oubliera pas de rappeler combien l'annotation des films dépend de conventions précises qui impliquent un certain regard du cinéma. Or, la mémoire populaire des films est une mémoire d'acteurs, d'acteurs américains quí «parlent français» puisque tous ces films étaient diffusés en version doublée. Parler «d'auteurs» de films ou «d'artistes» n'a aucun sens pour ces ouvriers; il serait donc plus juste de citer des acteurs, mais là aussi, l'entreprise risquerait de se révéler aussi vaine que peu probante. Des noms font certes l'unanimité: Humphrey Bogart, Audie Murphy, Alan Ladd, Jack Palance, Errol Flynn, Henry Fonda, John Wayne, Rita Hayworth, Jean Simmons, etc., mais il serait plus intéressant encore une fois d'en analyser les jugements.

L'usage de mots tels «chômeur», «garde réfectoire» ou 
«manœuvre», directement empruntés au vocabulaire de statuts professionnels officiels, servent ainsi à la constitution de catégories esthétiques de perception renvoyant respectivement des acteurs (et donc des films) au «non-cinéma» (comme lorsque l'on affirme que «tous les acteurs français sont des chômeurs»), à la nullité dramatique («garde réfectoire») ou au contre-emploi malheureux, voire à l'incompétence professionnelle («manœuvre»). Ces catégories jouent ainsi un rôle très important dans la logique d'une construction de la figure du héros: on met de l'avant par exemple Humphrey Bogart (un «sacré acteur comme on en fait plus») par opposition à Eddie Constantine, véritable «chômeur» du film «policier».

Le manœuvre («salarié affecté à des travaux ne nécessitant pas de connaissances professionnelles spéciales» — définition du Larousse) le plus enclin aux remarques soulignant le discrédit, désigne en fait celui qui, dépouғvu de qualifications spécifiques, est ramené à un travail à la fois «gauche» et «grossier», malgré le plus souvent la bonne volonté qui l'anime. Il se trouve là sans l'avoir demandé et fait le maximum pour dissimuler l'absence des qualités requises.

L'expression «manœuvre» sert ainsi à désigner toutes ces situations incongrues, d'imitations prétentieuses et ratées où les Français ont (systématiquement) 50 ans de retard sur les Américains. C'est par exemple Jean Marais dans des «films d'action» ou Yves Montand dans le rôle d'un boxeur (L'Idole d'Alexandre Esway, France, 1948).

De la plus dépréciative (le «chômeur») à la moins dépréciative (le «manœuvre»), ces insultes sont structurées autour du monde de l'usine, avec notamment la figure centrale du «garde réfectoire» (à la fois à l'intérieur et à l'extérieur de l'usine). Plus on s'éloigne du monde de l'usine vers le monde du dehors ("garde réfectoire», «chômeur»), plus la qualité attribuée est dévalorisante. Le «manœuvre» représente ainsi le degré zéro de la hiérarchie de l'usine, mais également l'élément le plus proche des autres objets de nature industrielle ordonnés selon le principe de justification de l' «efficacité» ${ }^{28}$. La juxtaposition des occurrences «chômeur»/ «manoeuvre» et la connotation négative qui leur est attribuée permettent de situer avec précision la genèse de telles expressions. 
Il est certain que le terme «chômeur» ainsi employé ne peut se concevoir qu'à une période (l'immédiat après-guerre) où le plein emploi renvoyait l'absence d'activité professionnelle à l'expression d'une «mauvaise volonté» ou à la traduction d'une inaptitude fondamentale à l'habileté technique, fût-elle employée au service d'un travail peu qualifié et répétitif.

La logique industrielle mise en œuvre ne doit pas être cependant entendue comme un simple reflet de la réalité ou comme le dévoilement d'une homologie de «perception» entre des produits d'usine et des produits cinématographiques de la part d'ouvriers sidérurgistes. On retomberait alors à nouveau dans une forme de déterminisme vulgaire. Les paroles du «monde industriel» sont utilisées comme des «catégories» bonnes à penser le cinéma ${ }^{29}$. Elles n'ont de sens que dans la mesure où elles servent à appréhender des univers qui n'ont justement rien de commun avec l'usine. Les classifications opérées à propos des films ne sont donc pas le résultat de comportements déterminés par les mêmes conditions sociales d'existence, mais par la même expérience esthétique. C'est la longue familiarité avec le cinéma qui explique la capacité pour certains ouvriers à exprimer un ensemble de jugements cohérents, un code de lecture autonome caractéristique d'une véritable «cinéphilie ouvrière».

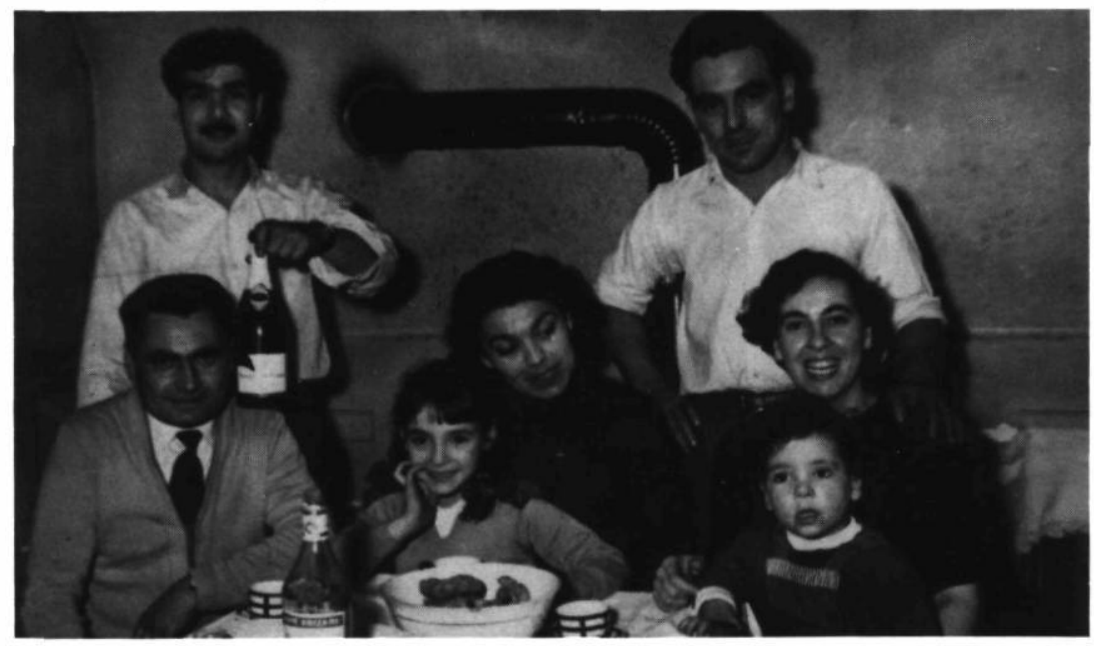

Une famille ouvrière d'origine italienne à Longwy en 1955 Photo: M. Leveratto 
Dans les années 40-50, à Longwy, un code de lecture «populaire», transmis oralement et reposant sur un savoir empirique, un raisonnement par analogies et une culture de "connaisseurs», s'est ainsi constitué parallèlement à la mise en place d'un code de lecture caractéristique de l'esthétique bourgeoise, déposé dans les livres et les manuels ou transmis par l'intermédiaire d'une disposition esthétique. Ce code particulier s'est d'autant plus facilement imposé au sein de fractions de la classe ouvrière locale, qu'il se vivait dans l'ignorance de stratégies de distinction supérieures et à un moment où les catégories esthétiques dominantes de perception des films n'étaient pas encore totalement constituées, ni établies.

Laffirmation de cette hypothèse pose alors quelques questions à l'histoire sociale. Ces «ouvriers cinéphiles», d'origine italienne, sont nés en France à la fin des années 20 et y ont été scolarisés. La vie urbaine et la sociabilité ouvrière, le passage par l'école (aussi bref soit-il) sont à l'origine de structures mentales nouvelles qui contrastent fortement avec celles de leurs parents (paysans immigrés, déracinés et analphabètes) et favorisent l'appropriation du cinéma. Ce dernier est d'ailleurs vécu sur le mode de la frénésie (cinq à six sorties par semaine) et alimente des jeux de distinctions subtiles entre ouvriers eux-mêmes, entre les «vrais amateurs de cinéma» et «ceux qui ne sont bons qu'à travailler comme des fous à l'usine». L'usage des mots «chômeur», "garde réfectoire», «manœuvre» reflète l'univers particulier d' «ouvriers spécialisés» ou "professionnels», par opposition à celui de la main d'œuvre non qualifiée, orientée vers les travaux les plus pénibles et ingrats, essentiellement confiés aux travailleurs immigrés (au début du siècle et dans les années 20, aux Italiens; après la Seconde Guerre mondiale, aux Maghrébins et aux Italiens du Sud ${ }^{30}$. Le code de lecture des films traduit, quant à lui, un univers esthétique aux antipodes de celui des cadres ou militants des principales organisations ouvrières de la région (PCF, CGT $)^{31}$. C'est pourtant à partir de ces ouvriers «italiens» de la deuxième génération que le PCF et la CGT sortiront de leur isolement au lendemain de la guerre et construiront leur lente hégémonie dans le bassin de Longwy, en y puisant les cadres, les militants, les adhérents, les sympathisants et les électeurs ${ }^{32}$. 
Au même moment, dans une commune située à la périphérie de Longwy, à quelques mètres des usines de Lorraine-Escaut, une salle de cinéma projette régulièrement des films égyptiens en langue arabe aux travailleurs maghrébins de la région. La pratique cinématographique de ces derniers, célibataires, jeunes et d'immigration récente, tranche avec celle des ouvriers du même âge, français ou nés en France de parents italiens. Cette fréquentation «mécanique», uniquement axée sur les films de langue arabe, sans «culture cinéphilique populaire», correspond plus à un réflexe de sociabilité du déracinement qu'à une pratique légitimée au sein de l'ensemble de la population ouvrière de la région. Elle témoigne également de la «non-intégration» relative de ces travailleurs au groupe ouvrier hégémonique (les Français et les Italiens de la deuxième génération).

Létude des usages populaires du cinéma révèle ainsi l'existence de micro-univers symboliques complexes, s'imbriquant les uns aux autres, sans pour autant constituer un ensemble homogène ou le reflet unique d'une classe ouvrière que l'on croit et pense uniforme.

\section{Conclusion}

Il faut bien évidemment se garder de voir, dans les catégories analytiques employées («principe de justification» et «code de lecture») la traduction de faits réels. Le chercheur reconstruit ainsi $a$ posteriori des grilles de lecture, à partir d'attitudes, de «rapports pratiques à la pratique ${ }^{33}{ }^{\text {» }}$, qui ne se sont jamais posés la question de leur objectivation. L'oralité qui caractérise jusqu'à présent toutes les formes de «cultures populaires» ne facilite guère la tâche de ceux qui se sont fixés pour but la recherche de sens dans ce que d'autres considèrent comme un amas inorganique d'idées. Cette labilité n'est pourtant pas une fatalité. En choisissant un terrain d'enquête, le cinéma, qui a rencontré autrefois ses faveurs auprès des classes populaires et s'est trouvé ainsi investi de formes particulières et autonomes de jugement, nous espérons pouvoir révéler mieux que nulle part ailleurs les principes qui commandent le rapport au monde et aux autres des ouvriers de Longwy, c'est-à-dire leur culture. Une histoire sociale du cinéma attentive à la mise en valeur des principes de justification et des 
codes de lectures de micro-groupes pourraient représenter une alternative au dilemme des études de «sociologie du cinéma» (entendue comme discipline visant à «établir des rapports d'homologie entre les films et-le milieu où ils naissent») condamnées selon Pierre Sorlin «à dire deux fois la même chose: ou bien décrire la société et vérifier la description dans les films, ou bien analyser les films et retrouver dans la structure sociale le schéma ainsi dégagé» (1977, p. 48).

Si la tâche paraît difficile, la question demeure: que pensaient donc ces gens des films qu'ils allaient voir dans la «caverne de voleurs», la salle de cinéma, dont parle Jean-Paul Sartre dans Les Mots?

\section{Institut Universitaire Européen de Florence (San Domenico di Fiesole)}

\section{NOTES}

1 Dans son dernier livre traduit en français, Francesco Casetti fait le point sur les théories du ou des spectateur(s) en les ramenant aux logiques internes propres à chaque discipline. Conscient des limites de telles approches et de la sienne, il appelle de ses vœux des «micro-histoires de la consommation». Voir Francesco Casetti, D'un regardl'autre, le film etson spectateur (Lyon: Presses Universitaires de Lyon, 1990).

2 La crise de la sidérurgie en 1978 a favorisé la publication d'un nombre considérable d'études sociologiques ou historiques sur le bassin industriel de Longwy. On se contentera de rappeler qu'il s'agit là d'une petite ville du nord de la Lorraine marquée par une grande concentration ouvrière et une forte immigration, notamment italienne, et on renverra le lecteur à la synthèse la plus importante réalisée à ce jour sur l'histoire du bassin: Gérard Noiriel, Longwy, immigrés et prolétaires, 1880-1980 (Paris: PUF, 1984). En 1946, le bassin de Longwy-Villerupt compte 52402 habitants dont 10897 étrangers (20,8\%). À la même époque, les usines sidérurgiques et les mines de fer de la région emploient près de 23000 personnes ( $20 \%$ d'Italiens, $10 \%$ de frontaliers belges, $9 \%$ d'Algériens). Bon nombre de Français sont aussi des étrangers récemment naturalisés (fin des années 30 et immédiat après-guerre). On compte également une vingtaine de salles de cinéma.

3 Pour une présentation de la micro-histoire, ses «protagonistes» et leur méthode, voir Jacques Revel, «L'Histoire au ras du sol», préface au livre de 
Giovanni Levi, Le Pouvoir au village (Paris: Gallimard, 1989) p. I-XXXIII. Outre le dépouillement de la presse locale et régionale, des bulletins paroissiaux, des parutions militantes, la lecture de nombreuses études historiques et sociologiques sur le bassin de Longwy-Villerupt, nombre de données ont été relevées à partir d'entretiens oraux semi-directifs et d'observations ethnographiques. Quinze personnes ont plus particulièrement été interrogées parmi lesquelles on trouve 11 hommes et 4 femmes, 8 anciens ouvriers (6 hommes, 2 femmes), les 7 autres personnes étant d'anciens employés, instituteur, professeur ou responsable syndical. La plupart sont nées à la fin des années 20. Douze sont françaises d'origine étrangère (11 d'origine italienne, 1 d'origine polonaise). Àl'exception d'une seule personne, elles ont toutes été scolarisées en France. Les interviews ontétésystématiquement retranscrites. De nombreux autres contacts ou discussions informelles n'ont pas fait l'objet d'enregistrement au magnétophone mais ont été consignés dans des annotations ultérieures. J'ai également fait appel à ma propre expérience d'étudiant issu d'un milieu ouvrier. Nombre d'hypothèses et d'intuitions ont été développées non seulement à partir de souvenirs personnels, mais aussi de situations où je ne faisais plus figure «d'étranger».

4 Voir Richard Hoggart, «Les Attitudes, les manières et les formes de sociabilité proprement populaire», La Culture du pauvre (Paris: Éditions de Minuit, 1970) p. 29. Il ne nous est pas possible, dans les limites qui nous sont imparties ici, de nous positionner par rapport au débat sur la «culture populaire». On se contentera d'indiquer quelques références, notamment Carlo Ginzburg, Le Fromage et les vers (Paris: Flammarion, 1980) p. 7 à 28 qui resitue bien le débat; Yves Lequin, «Jalons pour une histoire de la culture ouvrière en France», in Milieux, $\mathrm{n}^{\text {os }} 7 / 8$ (octobre 81-janvier 82) p. 70-79 pour la distinction entre "culture populaire» et "culture ouvrière»; Claude Grignon et Jean-Claude Passeron, Le Savant et le populaire (Paris: Hautes Études, Gallimard, Seuil, 1989) pour les questions de méthodologie; J. Clarke, C. Crichter et R. Johnson, Working-class culture in history and theory (Hutchinson, 1979, reprinted 1987) pour les études britanniques; Chandra Mukerji et Michael Schudson, «Popular Culture», Annual Review of Sociology, vol. 12 (1986) p. 47-66 (traduction française in Politix, «La Culture populaire, repères bibliographiques», $\mathrm{n}^{\circ} 13$, $\mathrm{p}$. 20-34) pour de larges indications bibliographiques.

5 Dès 1916, un étudiant de l'université d'Iowa s'inquiète du développement du cinéma considéré comme «un puissant facteur de façonnement des esprits et des mœurs d'une nation» (A socialstudy of the Motion Picture, cité par Garth Jowett, Film, The democratic art (Boston-Toronto: Little Brown and Company, 1976) p. 84). Des études plus anciennes encore sont signalées par Jean-Louis Leutrat, L'Alliance brisée, le western des années 1920 (Lyon: Presses Universitaires de Lyon, 1985) p. 45 et suivantes. Carl Vincent, Ricardo Redi et Franco Venturini recensent dans leur Bibliografia generale del cinema (Roma: Edizioni dell'Ateneo, 1953) près de 500 ouvrages dans un chapitre intitulé «les problèmes sociaux et moraux»; il s'agit des livres concernant «les rapports 
entre le cinéma et le public». Voir également Jean Mitry, Bibliographie internationale du cinéma et de la télévision, (première partie), France et pays de langue française, tome I (Paris: IDHEC, 1966); il recense 156 documents, articles et livres sous le paragraphe «sociologie».

6 Voir, par exemple, la violente dénonciation des «industries de biens culturels" par Max Horkheimer et Theodor Adorno, La Dialectique de la raison (Paris: Gallimard, 1974) p. 129 à 176 (traduction française de Dialektik der Aufklärung. Philosophische Fragmente (New York: Social Studies Association, 1944) et d'une certaine manière la prise de position opposée de Richard Hoggart, op. cit.

Les numéros spéciaux que certaines revues d'histoire ont, beaucoup plus récemment, consacrés au cinéma, ne font que reprendre, sans les critiquer, les postulats qui fondaient les anciennes approches, et notamment la question de l'«influence» ou l'«impact» des films sur «la masse» des spectateurs. Cf. Revue d'histoire Moderne et Contemporaine, "Cinéma et société» (avril-juin 1986); IlMovimento Operaioe Socialista, «Il Tempo dei media», $\mathrm{n}^{\circ} 2$, anno IX (1986); Journal of Contemporary History, «Historians and Movies, The State of Art», part 1 et part 2, vol. 18, $\mathrm{n}^{\circ} 3$ (déc. 1983) et vol. 19, $\mathrm{n}^{\circ} 1$ (janv. 1984); New German Critique, «Special Issue on Weimar Film Theory», $n^{\circ} 40$ (winter 1987).

La thématique de l'«aliénation» comprend celle du «divertissement» et en général toutes les pensées qui font du cinéma un refuge aux malheurs de la vie quotidienne ou aux contraintes sociales (l'équivalent de «la religion, opium du peuple» du jeune Marx, encore marqué par une philosophie idéaliste). Â aucun moment n'est envisagée la possibilité du cinéma comme un phénomène que l'on s'approprie et qui aide à la formation d'une vision du monde originale et spécifique, un acte aussi désintéressé et gratuit que la contemplation d'un tableau par un fin lettré, par exemple. Tous ces débats sont discutés dans mon mémoire de DEA, Hypothèses de recherche sur les usages populaires du cinéma (Paris: Université de Paris III, 1990).

7 Voir à ce sujet les discours des élites traditionnelles comme Barrès, Maurras, Ortega Y Gasset, Chesterton, Spengler, Keyserling, Klages et les points de vue opposés de Walter Benjamin, L'Oeuvre d'art à l'ère de sa reproductibilité technique (Paris, 1936, aujourd'hui in L'Homme, le langage et la culture (Paris: Denoël, 1971)) et Arnold Hauser, Histoire sociale de l'Art et de la Littérature, tome IV, l'époque contemporaine (Paris: Le Sycomore, 1984). Voir également Rik Stallaerts, «Glamour Rouge, les maisons du peuple et leurs cinémas», in "Pain noir et film nitrate, le mouvement ouvrier socialiste belge et le cinéma durant l'entre-deux-guerres», Revue belge du cinéma, $\mathrm{n}^{\circ} 15, \mathrm{p} .17$ et suivantes; Stephen G. Jones, The British labour movement and film, 19181939 (London: Routledge \& Reagan Paul Ltd, 1987).

8 Dans son dernier livre, Pierre Sorlin souligne avec justesse que «la réception 
est le point aveugle des études filmiques». Pierre Sorlin, European cinemas, European societies, 1939-1990 (London: Routledge, 1991) p. 10.

9 Ibid, p. 81-110.

10 Luc Boltanski, «Taxinomies populaires, taxinomies savantes: les objets de consommation et leur classement», Revue française de sociologie, $\mathrm{n}^{\circ} 1$ (janvier-mars 1970) p. 34.

11 Patricia Hubert-Lacombe, «L'Accueil des films américains en France pendant la guerre froide», Revued'Histoire Moderne et Contemporaine (avriljuin 1986) p. 301-315.

12 Richard Hoggart, op. cit. L'article stimulant de Steven J. Ross, «Struggles for the screen: workers, radicals, and the political uses of silent film», The American Historical Review, vol. 96, $\mathrm{n}^{\circ} 2$ (april 1991), part d'hypothèses qui nous sont très proches. Il repose cependant sur un postulat que nous ne pouvons partager: l'identification de la classe ouvrière aux organisations politiques et syndicales qui se sont fixées pour but de la représenter.

13 Cf. Fabrice Montebello, Le Cinéma à Longwy (1948-1953), essai de sociologie de l'ail populaire (Nancy: Université de Nancy II, 1989); Jeffrey Richards and Dorothy Sheridan, Mass-Observation at the movies. (London: Routledge \& Kegan Paul Ltd, 1987); Micheline Guaita, Le Public lyonnais face au cinéma 1895-1927, Thèse de $3^{\text {eme }}$ cycle en ethnologie (Lyon: Université de Lyon II, 1983); Jeffrey Richards, The Age of The Dream Palace, Cinema and Society in Britain 1930-1939 (London: Routledge \& Kegan Paul, 1984). Sur la géographie sociale des salles de cinéma dans une ville ouvrière, voir l'intervention de Brian Rigby, «Bolton and the cinema: from Mass-Observation to the "diaries of a nobody"», au colloque international de l'Université de Warwick, Popular European Cinema (University of Warwick, 1989). Une vue d'ensemble sur les salles de cinéma européennes se trouve dans la dernière synthèse de Pierre Sorlin, op. cit., p. 82-92. Voir également les deux études locales de Comunicazioni sociali sur la ville de Milan, «Il cinema a Milano tra le due guerre», a cura di Francesco Casetti e Raffaele De Berti, Comunicazioni sociali, 3-4, Anno X (luglio-dicembre 1988); «Il cinema a Milano dal secondo dopoguerra ai primi anni sessanta», a cura di Raffaele De Berti, Comunicazioni sociali, 1-2, Anno XIII (gennaio-giugno 1991), et la petite analyse sur les salles de cinéma américaines des années 30-40 in Colin MacCabe (ed.), High theory low culture, analysing popular television and film (Manchester: Manchester University Press, 1986). Pour le public ouvrier, voir le livre de Peter Stead, Film and the Working Class (London and New York: Routledge, 1989) chapitres 1,9 et 10 .

14 C. Vincent, R. Redi et F. Venturini, op. cit., p. 142. 
15 Pierre Bourdieu, La Distinction: critique sociale du jugement (Paris: Éditions de Minuit, 1979). Sur les études de sociologie de la culture en général, voir Raymond Williams, The sociology of culture (New York: Schoken books, 1981) (édition américaine de Culture, London, 1981) p. 9-32.

16 Fabrice Montebello, Le Cinéma à Longwy (1948-1953), essai de sociologie de l'œil populaire, op. cit., p. 107-108.

17 Ibid., p. 60-66 et 127-128. Cf. le rôle joué par une association comme «travail et culture» (Dudley Andrew, André Bazin, op. cit.).

18 Antoine de Baecque, Les Cahiers du cinéma, Histoire d'une revue, (tome I; à l'assaut du cinéma 1951-1959) (Paris: Éditions Cahiers du cinéma, 1991).

19 Pierre Bourdieu, La Distinction: critique sociale du jugement, op. cit.

20 C'est par ce type d'excès que la théorie de la légitimité culturelle rejoint la problématique de l'aliénation et tombe alors dans l'ethnocentrisme de classe (la tendance inverse du relativisme culturel étant de verser dans le populisme). Pour ces critiques et remarques à Pierre Bourdieu, voir Claude Grignon et JeanClaude Passeron, op. cit., p. 139.

21 Cette problématique ne fait qu'inverser celle de l'inférence des caractéristiques du spectateur à partir du film. Voir, par exemple, l'article de Patrick Champagne (rédigé sous la direction de Pierre Bourdieu), «La Télévision et son langage: l'influence des conditions sociales de réception sur le message», Revue française de sociologie, $\mathrm{n}^{\circ} 3$ (juillet-septembre 1971) p. 406-430.

22 Signalons cependant une position équivalente de Pierre Bourdieu dans un ouvrage plus récent. Cf. Pierre Bourdieu, Choses dites (Paris: Éditions de Minuit, 1987) p. 184.

23 Voir Fabrice Montebello, Le Cinéma à Longwy (1948-1953), essai de sociologie de l'ail populaire, op. cit.; Micheline Guaita, op. cit. Malgré un point de vue qui voit essentiellement dans le cinéma un moyen de supporter ou d'oublier la dure réalité de la vie quotidienne, Stephen G. Jones signale quelques études définissant le cinéma comme lieu de sociabilité et point de rencontre où peuvent prendre place «une vision collective du monde». Stephen G. Jones, op. cit., p. 12.

24 Howard S. Becker, Les Mondes de l'art (Paris, 1988).

25 La programmation de l'Acier Ciné-Club pour l'année 1953 est la suivante: Alexandre Nevsky, Citizen Kane, La Dernière Étape, Fantôme à vendre, La Bataille de l'eau lourde, La Beauté du diable, Le Silence est d'or, Les Enfants du paradis, Scinscià, Whisky à gogo et Les Roseaux du Lac Balaton. 
26 La Voix de l'Est (24 août 1946). La plupart de ces films sont soviétiques, en provenance des Pays de l'Est ou français marqués politiquement: Les 13, Nikita, Gloire à Moscou, Salut à Moscou, Cour d'Acier, Oural, Le Chant de la terre sibérienne, l'Arc-en-ciel, Djoulbars, Zoïa, Jeunesse radieuse, La Chute du tyran, Un homme véritable, La Vie est belle, Le Serment, La Marseillaise, etc.

27 Nous avons vérifié ce qui nous était souvent présenté comme un fait marquant, presque mythique, dans les enquêtes orales, en analysant minutieusement la programmation du Cinéma des Familles de 1948 à 1953. Les films américains représentent 70 à $90 \%$ de l'ensemble des longs métrages présentés. Ils attirent également en moyenne plus de spectateurs que les films des autres nationalités. Parmi les plus fortes entrées en une semaine de représentation (la salle contient 400 places), on trouve:

en 1948: Sauvagesse blanche (USA) - 1864 entrées — Le Mystère du temple vaudou (USA) - 2216 entrées

en 1949: Mabokl'éléphant du diable (USA) - 1979 entrées — Les Indomptés (USA) - 1936 entrées

en 1950: Tarzan et les sirènes (USA) - 1953 entrées

en 1951: Tarzan et la fontaine magique (USA) - 1589 entrées

en 1952: Captive parmi les fauves (USA) - 1542 entrées - Colt 45 (USA) - 1522 entrées

en 1953: Au Mépris des lois (USA) - 1599 entrées - Marqué au fer (USA) - 1550 entrées

28 Voir Fabrice Montebello, Hypothèses de recherche sur les usages populaires du cinéma, op. cit., p. 64-91.

29 Cf. Pierre Bourdieu, Roger Chartier et Robert Darnton, «Dialogue à propos de l'histoire culturelle», Actes de la rechercheen sciencessociales, $\mathrm{n}^{\circ}$ 59, p. 88.

30 La hiérarchie officielle dans les usines sidérurgiques de la région au lendemain de la Seconde Guerre mondiale est la suivante: OP ou «ouvrier professionnel» (OP1, OP2, OP3), OS ou «ouvrier spécialisé» (OS1 et OS2), MF ou «manœuvre de force» et MO ou «manœuvre ordinaire». En 1948, les OS représentent $46,5 \%$ de l'effectif ouvrier total devant les OP $(25,8 \%)$, les MF $(25 \%)$ et les MO (2,5\%). Cf. «Union des Mines et de la Métallurgie de Longwy», Longwy-Villerupt 1956 (Longwy, 1956) p. 70.

31 Voir Fabrice Montebello, Le Cinéma à Longwy (1948-1953), un essai de sociologie de l'ail populaire, op. cit., p. 118-120.

32 Cf. S. Bonnet, Ch. Santini, H. Barthelemy, «Appartenance politique et attitude religieuse dans l'émigration italienne en Lorraine sidérurgique», 
Archives de sociologie des religions, $\mathrm{n}^{\circ} 13$ (1962).

33 Pierre Bourdieu, Le Sens pratique, op. cit.

\section{OUVRAGES CITÉS}

Bourdieu, Pierre. La Distinction: critique sociale dujugement. Paris: Éditions de Minuit, 1979.

Bourdieu, Pierre. Le Sens pratique. Paris: Éditions de Minuit, 1980.

Grignon, Claude et Passeron, Jean-Claude. Le Savant et le populaire. Paris: Hautes Études, Gallimard, Seuil, 1989.

Sorlin, Pierre. Sociologie du cinéma. Paris: Aubier, 1977. 